\title{
Algunas dimensiones relacionadas con el rendimiento académico de estudiantes de Administración y Dirección de Empresas*
}

\author{
Some Aspects of the Business Administration \\ Students Academic Performance
}

Recibido: marzo 22 de 2012 | Revisado: octubre 28 de 2012 | Aceptado: diciembre 18 de 2012

\author{
Ma Valle Santos Álvarez** \\ ELEUTERIO VALLELADO *** \\ Universidad de Valladolid, España
}

doi:10.11144/Javeriana.UPSY12-3.adrr

Para citar este artículo: Santos, M. V. \& Vallelado, E. (2013). Algunas dimensiones relacionadas con el rendimiento académico de estudiantes de Administración y Dirección de Empresas. Universitas Psychologica, 12(3), 739-752. doi:10.11144/Javeriana. UPSY12-3.adrr

* Artículo de investigación. Eleuterio Vallelado ha recibido financiación para el desarrollo de la investigación del Ministerio de Ciencia e Innovación, proyecto: ECO2011-29144-C03-01. Los autores agradecen los comentarios de los evaluadores.

** Universidad de Valladolid, España. Profesora Titular del Departamento de Organización de Empresas. Researcher ID: H-6411-2013.E-mail: mvalle@ eco.uva.es

**** Universidad de Valladolid, España. Catedrático del Departamento de Economía Financiera y Contabilidad. Researcher ID: H-6297-2013.E-mail: teyo@ eco.uva.es
RES UMEN

En esta investigación se analiza el efecto de distintas variables sobre el rendimiento académico. Para esto se toma como referencia el modelo 3P (presagio-proceso-producto). El estudio empírico se desarrolla con alumnos universitarios. Los resultados indican que el rendimiento conceptual difiere significativamente del rendimiento procedimental. Se constata que la capacidad explicativa de las variables de presagio resulta superior a las variables de proceso, tanto en el rendimiento conceptual como en el procedimental. Además, el estilo cognitivo, el género y la asistencia resultan factores determinantes del rendimiento académico en todas sus dimensiones. Así, los estudiantes más analíticos, varones y con alto nivel de asistencia obtienen mejores resultados. En el rendimiento procedimental y global afecta también la motivación y el autoconcepto académico.

Palabras clave autores

Rendimiento conceptual, rendimiento global, rendimiento procedimental, variables presagio, variables proceso.

Palabras clave adicionales

Psicología educativa, administración de empresas, investigación cuantitativa.

\section{A B S T R A C T}

The goal is to analyze the determinant factors of each of the academic performance dimensions. The theoretical foundation is the 3P model (presage, process, and product). The empirical analysis is developed with students of Business administration in their last academic year. The results show that the conceptual performance is difference from the procedural performance and that such difference is statistically significant. The presage variables are more relevant that the process variables explaining either the conceptual performance or the procedural performance. Furthermore, the cognitive style, the gender and class attendance are explanatory factors of the academic performance in all the three dimensions. Male students with higher analytic capabilities and high attendance are the ones with higher performance. Student motivation and academic self-concept are variables that contribute to explain procedural and global performance.

Key words authors

Conceptual performance, procedural performance, global performance, presage variables, process variables.

Key words plus

Educational Psychology, Business Administration, Quantitative Research. 


\section{Introducción}

Actualmente estamos viviendo un momento de reflexión sobre el proceso de enseñanza-aprendizaje y los resultados que se derivan del mismo. En este sentido, la investigación reconoce el interés de analizar las distintas estrategias de aprendizaje desarrolladas por los alumnos y los distintos métodos de enseñanza implementados por los docentes. Además, el estudio del rendimiento académico de los alumnos constituye el punto de referencia para valorar el grado de eficacia del proceso de enseñanza-aprendizaje. El rendimiento académico se considera como el indicador del nivel de aprendizaje que ha alcanzado el estudiante al final de dicho proceso (Maquilón \& Hernández, 2011).

El nuevo contexto educativo propone una transición desde la "educación del enseñar" hacia la "educación del aprender" en la que el alumno es el elemento protagonista. En el proceso de acercamiento al alumno, el profesor, con frecuencia, combina distintas metodologías docentes (lección magistral, trabajos en grupo, trabajo autónomo del alumno, etc.) (Brown \& Atkins, 1988; Navarro, 2009). En la misma línea, el rendimiento académico es el resultado de la valoración que obtiene el alumno en distintos aspectos del proceso de enseñanzaaprendizaje: trabajos en grupo, exposiciones en clase, resolución de casos prácticos, demostración de conocimientos conceptuales de la materia, etc. La idea es enriquecer los procesos de evaluación y dar entrada a la variedad de capacidades implicadas en el conocimiento de una materia. Se pueden entonces identificar distintas dimensiones del rendimiento académico (De la Fuente, Pichardo, Justicia \& Berbén, 2008). Frecuentemente, los estudios disponibles recurren a un indicador único de rendimiento académico o a una media de los resultados obtenidos a lo largo de un periodo de tiempo (Echavarri, Godoy \& Olaz, 2007; Felman et al., 2008). Sin embargo, esta investigación reconoce el rendimiento académico como un constructo en el que convergen distintas dimensiones.

Por otro lado, la literatura apunta un amplio abanico de factores, tanto relacionados con el estudiante como con el contexto educativo, que afec- tan al rendimiento académico de los estudiantes (Corno \& Snow, 1986; De la Fuente et al., 2008; De la Fuente, Martínez, Peralta \& García, 2010; Echavarri et al., 2007; Feldman et al., 2008; García, Alvarado \& Jiménez, 2000; González-Pienda, Núñez Pérez, Glez.-Pumaniega \& García, 1997; Martín del Buey \& Camarero, 2001; Núñez et al., 1998; Rodríguez \& Herrera, 2009; Valle, González, Núñez \& González, 1998). Así, se señalan, entre otros, los siguientes: la motivación, el estrés académico, las propiedades cognitivas, el género, el nivel de autoconfianza, el apoyo social, el estilo de aprendizaje, las estrategias del aprendizaje, la metodología docente empleada, etc. Sin duda, todos ellos contribuyen, en mayor o menor medida, a la explicación de los diferentes niveles de rendimiento académico de los estudiantes. Ahora bien, ese conjunto abarca factores de diferente naturaleza: algunos son de índole individual que el alumno no puede modificar -por ejemplo, el género o los rasgos de personalidad-; otros pertenecen al alumno y sî los puede cambiar - por ejemplo, su estrategia de aprendizaje - y otros están vinculados al contexto educativo y, por tanto, se escapan del ámbito de influencia del alumno y afectan por igual a todos en el grupo. El modelo 3P (Presagio-Proceso-Producto) de Biggs (2001) constituye la estructura conceptual de referencia que permite estructurar las distintas variables que condicionan el rendimiento académico.

Con este punto de partida se plantean varias cuestiones. En primer lugar, se analiza si efectivamente en el rendimiento académico se pueden identificar distintas dimensiones o si todas ellas resultan coincidentes. Por otro lado, habrá que estudiar cuáles son las variables concretas, tanto de presagio como de proceso, que determinan el rendimiento académico. Por tanto, el siguiente objetivo de la investigación es identificar las variables de presagio y proceso que permiten explicar el rendimiento académico. Además, si, como se apuntó anteriormente, en el rendimiento académico convergen distintas dimensiones habrá que examinar por separado cada una de ellas. Esto plantea, a su vez, otra cuestión: las distintas dimensiones del rendimiento académico comparten la misma de- 


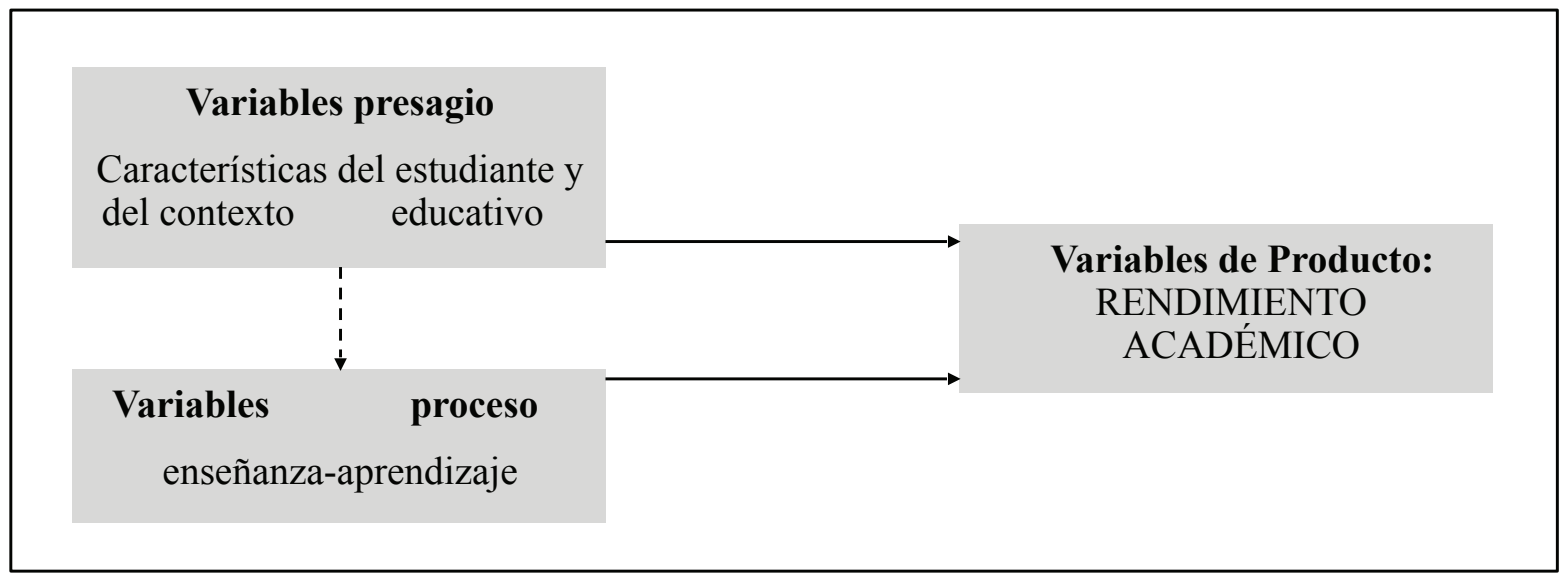

Figura-1: Modelo de análisis del rendimiento académico.

Fuente: elaboración propia.

pendencia con las variables de presagio y proceso o, por el contrario, cada una de las dimensiones muestra un perfil específico? Así, el siguiente objetivo de la investigación se dirige a analizar y comparar el conjunto de variables determinantes de las distintas dimensiones del rendimiento académico.

En suma, el propósito de la investigación se dirige a investigar las variables que condicionan el rendimiento académico en las distintas dimensiones que lo componen. Para esto, la investigación se estructura en distintas secciones. En la primera, se desarrolla el modelo de análisis y los elementos que la componen. En la segunda, se plantea la aplicación empírica del estudio y en la tercera se recogen los principales resultados. El estudio empírico se desarrolla con los alumnos de la Universidad de Valladolid (España) de la licenciatura de Administración y Dirección de Empresas en el curso 2010-2011. Finalmente, el estudio se cierra con las principales conclusiones e implicaciones que se desprenden de la investigación.

\section{Rendimiento académico: factores determinantes}

El rendimiento académico representa el nivel de aprendizaje del alumno, fruto del proceso enseñanza-aprendizaje. En la explicación de este concepto, la literatura reconoce la influencia de factores de diversa naturaleza. El modelo 3P (Biggs, 1988, 2001;
De la Fuente et al., 2010; Maquilón \& Hernández, 2011) proporciona un marco general de referencia para explicar el rendimiento académico, recogiendo tres grupos de variables: variables de presagio (características del estudiante y del contexto previo a la acción educativa), variables de proceso (dinámica enseñanza-aprendizaje) y variables de producto (satisfacción con el aprendizaje y rendimiento). El argumento básico indica que las variables presagio condicionan el proceso de enseñanza-aprendizaje que a su vez determinan el resultado final (Figura 1).

Las variables de resultado aluden a la satisfacción del alumno con el proceso de aprendizaje y el rendimiento académico. Este último se define como la productividad del sujeto, el producto final de la aplicación de su esfuerzo, matizado por sus actividades, rasgos y la percepción más o menos correcta de los cometidos asignados. Aunque en numerosos estudios el rendimiento académico se analiza de forma global (García et al., 2000; Rodríguez \& Herrera, 2009), en otros estudios se reconoce que en él se integran diferentes dimensiones (De la Fuente et al., 2008). En concreto, se distingue entre rendimiento conceptual-vinculado a la valoración de los contenidos conceptuales adquiridos por el alumno-, rendimiento procedimental-referido a la capacidad del alumno de ejecución y resolución de problemas relacionados con la materia- y rendimiento actitudinal -referido a las intervenciones del alumno en actividades voluntarias propuestas en el desarrollo de la materia-. 


\section{Variables de presagio}

Aluden a aquellas variables independientes del proceso de enseñanza, y relacionadas con las características personales del alumno y del contexto del proceso de aprendizaje. Este estudio se centra únicamente en variables individuales de los alumnos e incluye aquellas que a lo largo de la literatura se muestran relevantes en la explicación del rendimiento académico. En concreto, se incluyen cuatro bloques: cognitivas, de motivación, de personalidad y de género. Tradicionalmente, se ha reconocido que los factores de cognición y motivación operan conjuntamente para crear las condiciones óptimas de aprendizaje y rendimiento académico (González-Pienda et al., 1997; Núñez et al., 1998). Las variables cognitivas se refieren al marco individual en el que el alumno procesa y analiza información y viene representado por dos de ellas: estilo cognitivo y proactividad. El primero se refiere al proceso individual de procesamiento de información y, por ello, influye en las preferencias para distintos tipos de aprendizaje. La segunda alude a las diferencias individuales en cuanto a las acciones que realiza el individuo para influir y cambiar el entorno (Bateman \& Crant, 1993; Cools \& Van den Breek, 2008; Crant, 2000). La personalidad proactiva es aquella que busca oportunidades, muestra iniciativa, emprende acciones y persevera en su comportamiento hasta alcanzar el objetivo establecido (Bateman \& Crant, 1993; Cools \& Van den Breeck, 2008).

La motivación se refiere al conjunto de procesos implicados en la activación, dirección y persistencia de la conducta (Beltrán, 1993; Maquilón \& Hernández, 2011; McClelland, 1989). El análisis de la motivación individual toma como referencia la Regulatory Focus Theory de Higgins (1998), que analiza la forma en que los individuos enlazan sus tareas, objetivos y tendencias motivacionales. En concreto, reconoce dos orientaciones motivacionales: orientación promoción y orientación prevención. La orientación promoción es la que conduce a los individuos a preocuparse por el avance y el éxito y está relacionada con una actitud de apertura al cambio, ideales fuertes y asunción de riesgos. La orientación prevención se relaciona con la preocupación por la seguridad, la estabilidad y el cumplimiento de las obligaciones. Los individuos con mayor orientación promoción intentan alcanzar objetivos establecidos personalmente (motivación intrínseca), mientras que los individuos dominados por la orientación prevención persiguen objetivos más vinculados con el deber y la responsabilidad profesional (motivación extrínseca). Investigaciones previas relacionan motivación y enfoques de aprendizaje. Concretamente, la motivación intrínseca se asocia a altos logros educativos por parte de los estudiantes y a la utilización del enfoque profundo de aprendizaje (Maquilón \& Hernández, 2011).

La personalidad hace referencia a la tendencia del individuo para comportarse, pensar o sentir de manera determinada en diferentes situaciones a lo largo de su vida (Navarro, 2009). La investigación indica que los rasgos de personalidad median en la adquisición de competencias y, por tanto, constituyen factores determinantes del rendimiento académico (Navarro, 2009). Además, determinan las estrategias de los estudiantes universitarios para enfrentar el estrés (Contreras-Torres, EspinosaMéndez \& Esguerra-Pérez, 2009). El modelo de los Cinco Grandes (Big Five) agrupa las características de personalidad en cinco rasgos (Costa \& McCrae, 1992; Soto et al., 2011): extraversión, amabilidad, responsabilidad, neuroticismo y apertura a la experiencia. Los dos primeros hacen referencia a las relaciones interpersonales, el tercero a la orientación del individuo a la tarea y los dos últimos a las experiencias emocionales y cognitivas (Navarro, 2009). Este modelo se considera universal puesto que se ha hallado la misma estructura en diversas culturas (Contreras-Torres et al., 2009; Costa \& McCrae, 1992, 1999) y en distintos niveles de edad (John, Caspi, Robins, Moffittt \& StouthamerLoeber, 1994; Navarro, 2009).

Respecto al género, estudios previos indican que está relacionado con diferencias significativas en habilidades cognitivas (Echavarri et al., 2007) y en la utilización de estrategias y estilos de aprendizaje (Alonso, Gallego \& Honey, 1994; Amelang \& Bartussek, 1991; Cano, 2000; Martín del Buey \& 
Camarero, 2001). Las actividades que realizan los alumnos requieren de la utilización de estrategias y estilos de aprendizaje. Por tanto, la influencia del género en el rendimiento académico deviene no solo de diferencias en las capacidades cognitivas o intelectuales atribuibles al género, sino también de diferencias en las actividades de alumnos y alumnas en el seguimiento de la materia. En síntesis, se ha constatado que las alumnas tienden a adoptar con más frecuencia un enfoque superficial (Severiens \& Ten Dam, 1994) y los alumnos un estilo de procesamiento profundo, aunque los resultados son contingentes al tipo de carrera universitaria cursada (Cano, 2000). Asimismo, se ha constatado que las mujeres aventajan a los varones en pruebas de fluidez verbal y muestran menor dominio del razonamiento abstracto y cálculo (Echavarri et al., 2007).

\section{Variables de proceso}

Las variables de proceso están referidas a la dinámica enseñanza-aprendizaje, específicamente, a las actividades que realiza el alumno para desarrollar el proceso de aprendizaje de la materia y, por tanto, viene condicionado por el tipo de materia, la metodología docente, el estilo de enseñanza adoptado por el profesor, etc. En este estudio, las variables utilizadas para representar el seguimiento que realiza el alumno son las siguientes: nivel de asistencia, participación en clase y el autoconcepto académico del alumno en la materia. Estudios previos señalan que la asistencia y participación del alumno son relevantes en la predicción del rendimiento académico universitario (Alvarado \& García Jiménez, 1997; Álvarez, García \& Gil, 1999; Fernández, 2001; García et al., 2000; Rodríguez \& Herrera 2009); representan la motivación e interés del alumno hacia la materia y el proceso de enseñanza-aprendizaje, lo que justifica su influencia en el rendimiento académico.

El autoconcepto académico es un componente más del autoconcepto general, que constituye una fuente de motivación que influye directa y significativamente sobre los logros y las expectativas escolares del alumno (González-Pienda et al., 1997;
Santana, Feliciano \& Jiménez, 2009); ejerce un papel destacado en la regulación de las estrategias cognitivo-motivacionales implicadas en el aprendizaje y el rendimiento académico (Bandura, 1987). La implicación activa del sujeto en el proceso de aprendizaje aumenta cuando se siente autocompetente, es decir, cuando confía en sus propias capacidades y tiene altas expectativas de autoeficacia (González-Pienda et al., 1997). Se ha comprobado que el autoconcepto positivo favorece la utilización de procedimientos estratégicos de aprendizaje, lo que facilita al alumno un procesamiento profundo de la información (Núñez et al., 1998) y afecta su rendimiento académico. En concreto, las investigaciones confirman la relación entre la imagen que el alumno tiene de sí mismo y su implicación activa en el aprendizaje y la resolución de tareas académicas (Núñez et al., 1998).

En este estudio, el autoconcepto del alumno en la materia viene representado por la posición que ocupa el alumno en el aula. Concretamente, se entiende que aquellos alumnos que se sitúan en posiciones delanteras del aula son alumnos con mayor confianza en su capacidad para interactuar con el profesor y, por tanto, con mejor autoconcepto en la materia que aquellos que se sitúan en las filas traseras del aula.

\section{Método}

\section{Participantes}

Para la aplicación empírica se tomó como referencia el grupo de alumnos de la licenciatura de Administración y Dirección de Empresas (Universidad de Valladolid) que cursaban la asignatura Inversiones Financieras, durante el curso académico 2010-2011. Se trata de una asignatura obligatoria de 5. ${ }^{\mathrm{o}}$ curso (primer cuatrimestre), de seis créditos, dedicada al estudio de las decisiones de inversión en activos financieros. El grupo de análisis estuvo compuesto por 115 estudiantes de los cuales 49 eran chicos y 66 chicas. Los alumnos participaron de forma voluntaria y la muestra obtenida fue tratada conforme a las normativas internacionales de principios éticos. 


\section{Instrumentos}

Para la realización del estudio se solicitó la colaboración de los alumnos. Se les pidió que respondieran una serie de cuestionarios dirigidos a retratar su perfil cognitivo, motivación y rasgos de personalidad.

- Estilo cognitivo

Se utilizó el Índice de Estilo Cognitivo (CSI) que distingue dos categorías: intuitivo y analítico (Allinson \& Hayes, 1996). El individuo de estilo analista se caracteriza por estudiar detalladamente los problemas y adoptar decisiones sobre la base del razonamiento mental. El individuo de estilo intuitivo toma decisiones y aborda los problemas sobre la base del sentimiento. El cuestionario de CSI de Allinson y Hayes (1996) consta de 38 ítems que el participante debe puntuar en una escala de tres puntos ${ }^{1}$ (ciertos, indiferentes ofalsos). Por tanto, agregando las puntuaciones, el indicador individual de CSI va de 0 a 76 , aunque para el desarrollo del análisis las puntuaciones individuales se transportan a una escala 0-1. Puntuaciones elevadas se identifican con individuos de estilo analítico y las bajas representan individuos intuitivos. Se comprobó también que la escala cumple con el criterio de fiabilidad (el alfa de Cronbach alcanzó el valor de 0.89).

\section{- Proactividad (PRO)}

Se aplicó una escala de 10 ítems que se puntúan en una escala de 1 a 5 en función del grado de acuerdo (Santos, García \& Vallelado, 2010; Seibert, Kraimer \& Crant, 2001). Puntuaciones más altas reflejan mayor nivel de proactividad individual. Se comprobó que la consistencia interna de este indicador muestra un nivel satisfactorio (el alfa de Cronbach alcanzó el valor de 0.75).

\section{- Personalidad}

Se aplica el cuestionario Big Five que recoge 43 ítems acerca de las cinco dimensiones indicadas previamente: extraversión (EXT), amabilidad

1 La puntuación de las respuestas difiere si el ítem está relacionado con el estilo analítico o con el intuitivo. En el primer caso, las respuestas se puntúan con 2, 1, 0 si se califican como ciertas, indiferentes o falsas, respectivamente. En el caso de que el ítem se refiera al estilo intuitivo, las respuestas se puntúan con $0,1 \mathrm{o}$ 2 si se califican como ciertas, indiferentes o falsas.
(AGR), responsabilidad (CO), neuroticismo (NER) y apertura a la experiencia $(\mathrm{OP})$. Se comprueba que en todos los rasgos el alfa de Cronbach alcanza un valor satisfactorio ${ }^{2}$.

- Motivación

Se utilizó la escala de Arnold y Reynolds (2009), derivada de Semin, Higgins, Gil de Montes, Estourget y Valencia (2005), que incluye seis ítems referidos a la motivación prevención (PV) y seis ítems referidos a la motivación promoción (PM). En ambos casos la consistencia interna de la escala alcanza un nivel satisfactorio (PM-alfa de Cronbach $=0.84 ;$ PV-alfa de Cronbach $=0.83$ ).

Para completar la información se recoge también la plantilla de registro que cumplimentó el profesor en cada una de las clases. Esta plantilla registra la asistencia del alumno, su posición en el aula y, si corresponde, su participación activa. La asistencia se representa con la variable ASIST que mide en el intervalo 0-1 el porcentaje de clases a las que ha asistido el alumno durante el curso académico. En la misma línea, la variable representativa de la participación, PARTIC, recoge también, en el intervalo 0-1, la puntuación del alumno en la realización de trabajos voluntarios en la asignatura.

La posición en el aula se mide con una variable cualitativa que toma tres valores: 1) si el alumno se sitúa en las dos filas delanteras del aula, 2) si lo hace en las filas centrales del aula y 3) si se ubica en las dos últimas. Se dispone así de la posición del alumno en el aula en cada una de las clases. Después, se calcula la media con objeto de disponer de un indicador promedio de la posición del alumno (POSIC) ${ }^{3}$.

Para el análisis del rendimiento se reúne información referida al rendimiento conceptual y procedimental. Además se recoge el rendimiento académico global del alumno:

- Rendimiento conceptual (RC)

2 En todos los rasgos, excepto en el referido a neuroticismo, el valor del alfa de Cronbach supera el valor de 0.75 .

3 A pesar de la naturaleza cualitativa de la variable se calcula el valor medio con objeto de contar con una medida de la tendencia del alumno a la hora de situarse en el aula a lo largo de las distintas sesiones de la asignatura. 
Viene representado por la calificación del alumno en la parte del examen referida a contenidos conceptuales. Se trata de la puntuación que obtiene el alumno en una batería de cuestiones tipo test sobre el contenido teórico de la asignatura.

- Rendimiento procedimental (RP)

Para representarlo se recurre a la calificación obtenida por el alumno en la parte del examen referida a la práctica. Se trata de la resolución de casos representativos de situaciones cuasi-reales de inversión financiera.

- Rendimiento académico global (RAG)

Se refiere a la calificación obtenida por el alumno en el conjunto de la asignatura, que se deriva de la integración del rendimiento conceptual y procedimental.

\section{Procedimiento}

En octubre del 2010, se diseñó el estudio y se organizó el proceso de recogida de información en tres fases. En la primera, a mediados del mes de octubre, se contactó a los alumnos a quienes se les propuso la participación voluntaria en el mismo. Se les explicó el propósito del estudio y se les entregaron las encuestas dirigidas a representar el estilo cognitivo, la proactividad, la personalidad y la motivación. En la segunda fase, que abarcó todo el periodo de la asignatura; el profesor rellenó de manera puntual en cada una de las clases, la plantilla de registro en la que se anota la asistencia del alumno, la participación y la posición en el aula. En la tercera fase, al final del periodo de docencia de la asignatura, se recopiló la información referida a las distintas dimensiones del rendimiento académico.

\section{Análisis estadístico}

La base de datos se construyó en Excel y se importó a SPSS para su análisis estadístico. Con la información recopilada se procedió a abordar los objetivos de la investigación. En primer lugar, se analizó la existencia de diferencias en las dimensiones del rendimiento académico consideradas. En segundo lugar, se analizaron las variables propuestas como variables explicativas. A la luz de la estadística descriptiva se examinó el comportamiento de esas variables. Además se analizó la relación entre las variables explicativas con objeto de evitar solapamientos de información y problemas de multicolinealidad en la estimación. Finalmente, se examinó la capacidad de las variables propuestas para explicar el rendimiento académico de los alumnos. Con ese propósito, se planteó un modelo de regresión lineal por capas. En concreto, se estimaron tres modelos, uno por cada una de las dimensiones de rendimiento consideradas (conceptual, procedimental y global). Respecto a las capas del modelo, estas se correspondieron con las variables de presagio y de proceso, respectivamente.

\section{Resultados}

\section{Dimensiones del rendimiento académico}

En el estudio realizado el rendimiento académico distingue entre rendimiento conceptual, rendimiento procedimental y rendimiento global. Los resultados obtenidos indican que el rendimiento procedimental es el que alcanza los valores medios más bajos (4.49 frente a 5.45 en el conceptual y 5.51 en el global) y además muestran la mayor variabilidad (2.63). El rendimiento conceptual es el que muestra la menor variabilidad (1.7) y el rendimiento global es el que muestra el valor medio más alto (5.51). Por otro lado, el análisis de diferencia de medias constata que el rendimiento conceptual y el procedimental resultan significativamente diferentes entre sí ( $t=4.73$, Sig. < 0.001). Se constata entonces que en la muestra analizada, los alumnos obtienen puntuaciones significativamente diferentes en el rendimiento conceptual respecto del rendimiento procedimental. Por otro lado, el resultado del test de los signos indica que el rendimiento conceptual no solo difiere del rendimiento procedimental, sino que además es significativamente superior $(Z=-4.35$, Sig. $<0.001)$ a los niveles de confianza más exigentes. Se deduce entonces que estas dos dimensiones del rendimiento consiguen efectivamente valorar aspectos diferentes del proceso de aprendizaje del 
TABLA 1

Estadística descriptiva y correlación de variables de presagio

\begin{tabular}{lcccccccccccc}
\hline & \multicolumn{1}{c}{ Estadísticos } & \multicolumn{1}{c}{ Correlaciones } \\
\hline & M & DE & CSI & PRO & PM & PV & SEX & EXT & AGR & CO & NER & OP \\
CSI & 00.66 & 00.13 & 1 & .0 & -0.01 & $-0.28^{* *}$ & 0.11 & -0.13 & -0.07 & 0.01 & 0.02 & -0.18 \\
PRO & 00.74 & 00.11 & & 1 & $0.58^{* *}$ & $0.19^{*}$ & -0.11 & $0.25^{*}$ & 00.08 & 0.07 & 0.07 & $0.22^{*}$ \\
PM & 30.87 & 00.41 & & & 1 & $0.16^{*}$ & -0.06 & 0.19 & 00.06 & 0.16 & 0.11 & $0.23^{*}$ \\
PV & 20.87 & 00.6 & & & & 1 & $-0.28^{* *}$ & -0 & -0.05 & -0.05 & 0.06 & 0.03 \\
SEXO & 00.6 & 00.49 & & & & & 1 & 0.14 & $0.27^{* *}$ & 0.18 & $0.21^{*}$ & 0.05 \\
EXT & 00.59 & 00.19 & & & & & & 1 & $00.66^{* *}$ & $0.55^{* *}$ & $0.23^{*}$ & $0.6^{* *}$ \\
AGR & 00.69 & 00.17 & & & & & & & 1 & $0.71^{* *}$ & $0.43^{* *}$ & $0.62^{* *}$ \\
CO & 00.7 & 00.18 & & & & & & & & 1 & $0.46^{* *}$ & $0.68^{* *}$ \\
NER & 00.44 & 00.19 & & & & & & & & & 1 & $0.32^{* *}$ \\
OP & 00.6 & 00.16 & & & & & & & & & & \\
\hline
\end{tabular}

$* p<0.05 . * p<0.01$.

Nota: Estilo cognitivo (CSI), proactividad (PRO), motivación promoción (PM), motivación prevención (PV), extraversión (EXT), amabilidad (AGR), responsabilidad (CO), neuroticismo (NER) y apertura a la experiencia (OP).

Fuente: elaboración propia.

TABLA 2

Estadística descriptiva y correlación de variables de proceso

\begin{tabular}{lccccc}
\hline & \multicolumn{2}{c}{ Estadísticos } & \multicolumn{3}{c}{ Correlaciones } \\
\hline & $M$ & MD & PARTIC & ASIST & POSIC \\
PARTIC & 0.71 & 0.18 & 1 & $0.74^{* *}$ & 0.11 \\
ASIST & 0.78 & 0.26 & & 1 & -0.9 \\
POSIC & 1.87 & 0.6 & & & 1 \\
\hline
\end{tabular}

$* p<0.05 . * * p<0.01$.

Nota: participación (PARTIC), asistencia (ASIST) y posición en el aula (POSIC).

Fuente: elaboración propia.

alumno. Así, el proceso de aprendizaje abarca tanto la asimilación de conocimientos teóricos propios de la materia como la habilidad de aplicar esos conocimientos en la resolución de casos prácticos. Asimismo, los resultados revelan que los alumnos se muestran más eficaces en la asimilación de los contenidos conceptuales que en su aplicación. Estos resultados indican que la resolución de los casos prácticos requiere no solo la asimilación de conocimientos teóricos de la materia, sino también la capacidad de relacionarlos y trasladarlos a casos concretos.

\section{Análisis de las variables explicativas}

A continuación se analiza el comportamiento de las variables propuestas como explicativas del ren- dimiento. Además se examinan las relaciones entre ellas con objeto de detectar y eliminar variables redundantes en la estimación posterior. La estadística descriptiva de las variables de presagio y los coeficientes de correlación aparecen a continuación (Tabla 1).

Los resultados obtenidos indican que los estudiantes analizados se muestran más analíticos que intuitivos, notablemente proactivos y con una motivación promoción superior a la prevención, es decir en los que domina la motivación intrínseca. En cuanto los rasgos de personalidad, el indicador de neuroticismo no alcanza el valor de 0.5 , salvo éste, todos los demás alcanzan valores altos, lo que denota una cierta intensidad en esas características. Respecto al género, ya se ha apuntado que en la muestra hay mayoría de alumnas, por ello el valor 
medio supera el valor de 0.5. Se constata además, entre las variables de presagio, que los valores de la motivación prevención son los que muestran mayor variabilidad. Los coeficientes de correlación revelan que los rasgos de personalidad mantienen elevadas correlaciones positivas entre sí. Por tanto, de las cinco variables recogidas tan solo se incluirá una en la estimación de los modelos, la representativa de la extraversión (EXT), indicativa también de la capacidad del alumno de mantener relaciones personales. Además, el indicador del nivel de proactividad se revela redundante con las variables representativas de la motivación prevención y promoción. Por ese motivo la variable proactividad es eliminada de la estimación. También la variable representativa de orientación motivacional de prevención mantiene relaciones significativas y negativas con el indicador de estilo cognitivo (CSI) y con el género. Se deduce entonces que, en la muestra analizada, aquellos estudiantes con mayor nivel de motivación prevención son los más intuitivos y muy probablemente de género masculino. Se constata además que las dos variables de motivación mantienen correlación positiva y significativa entre ellas. La variable representativa de la orientación prevención, igual que en los casos anteriores, es eliminada del análisis.

A continuación se recoge la estadística descriptiva de las variables de proceso y sus coeficientes de correlación (Tabla 2).
En cuanto a las variables de proceso, los resultados indican que los alumnos de la muestra presentan elevados niveles de asistencia y participación y su posición se sitúa más hacia posiciones delanteras que en la parte trasera del aula. En esta variable, se encuentran también los niveles más elevados de variabilidad, y se constata que la representativa de la asistencia resulta redundante con la representativa de la participación activa en clase. Por esto, la variable indicativa de la participación es eliminada.

Suprimidas las variables redundantes, los dos bloques de variables explicativas del rendimiento académico se componen de las siguientes:

- Variables de presagio: índice de estilo cognitivo (CSI), motivación-promoción (PM), género (SEX) y extraversión (EXT).

- Variables de proceso: asistencia (ASIST) y posición en el aula (POSIC).

\section{Análisis de regresión}

Delimitado ya el conjunto de variables de presagio y proceso, se comprueba que entre ellas no mantienen relaciones relevantes que pudieran distorsionar la estimación de los modelos de regresión (Apéndice). Como se señaló anteriormente, a continuación se estima un modelo de regresión lineal por capas, incluyendo la primera las variables de presagio y la segunda, las de proceso. Los resultados obtenidos

\section{TABLA 3}

Resultados de la estimación de las variables de rendimiento académico a partir de las variables de presagio y de proceso

\begin{tabular}{lcccc}
\hline & & $\begin{array}{c}\text { RC: } \text { Rendimiento } \\
\text { Conceptual }\end{array}$ & $\begin{array}{c}\text { RP: Rendimiento } \\
\text { Procedimental }\end{array}$ & $\begin{array}{c}\text { RAG: Rendimiento } \\
\text { Global }\end{array}$ \\
\hline 1a capa: v. presagio & R2 Ajusta. & 0.08 & 0.109 & 0.104 \\
& F (Sig.) & $3.219(0.016)$ & $4.13(0)$ & $3.95(0)$ \\
$2^{\text {a }}$ capa: v. proceso & R2 Ajusta. & 0.138 & 0.172 & 0.22 \\
& F (Sig.) & $3.729(0.002)$ & $4.53(0)$ & $5.79(0)$ \\
& & CSI: $2.49(0.04)$ & CSI: $5.3(0)$ & CSI: $3.9(0.01)$ \\
& & - & PM: $1(0.07)$ & PM: $1(0.03)$ \\
V. significativas* & SEX: $-1.1(0)$ & SEX: $-1.3(0.01)$ & SEX: $-1.3(0)$ \\
& & ASIST: $1.4(0.01)$ & ASIST: $1.7(0.05)$ & ASIST: $2.3(0)$ \\
& & - & POSIC: $-0.8(0.03)$ & POSIC: $-0.8(0.01)$ \\
\hline
\end{tabular}

Nota. Presagio: índice de estilo cognitivo (CSI), motivación-promoción (PM), género (SEX) y extraversión (EXT). Proceso: asistencia (ASIST) y posición en el aula (POSIC).

* Variables significativas a niveles de confianza igual o superior al $95 \%$.

Fuente: elaboración propia. 
en la estimación de los modelos aparecen a continuación (Tabla 3).

Los resultados obtenidos revelan la desigual capacidad explicativa de las variables presagio y proceso en las distintas dimensiones de rendimiento. La capacidad explicativa de las variables de presagio resulta superior a las de proceso tanto a la hora de explicar el rendimiento conceptual como procedimental. Sin embargo, en el caso del rendimiento global, la capacidad explicativa de las variables de proceso resulta superior a la que proporcionan las de presagio. Además, la estimación del modelo de rendimiento global es el que alcanza una mayor capacidad explicativa (22\%). No obstante los tres modelos proporcionan estimaciones significativas.

Las estimaciones obtenidas permiten identificar los factores determinantes de las distintas dimensiones del rendimiento académico. El estilo cognitivo, el sexo y la asistencia resultan factores determinantes del rendimiento académico en todas sus dimensiones. Los estudiantes más analíticos, de género masculino y con alto nivel de asistencia son los que mejores resultados obtienen tanto a nivel conceptual, como procedimental y global. Sin embargo, en el rendimiento procedimental y global afectan de forma significativa además otras dos variables: la orientación promoción (motivación intrínseca) y la posición del estudiante en el aula. Los alumnos con mayor motivación promoción y situados en posiciones delanteras del aula son los que obtienen mayores rendimientos procedimentales y globales. La motivación promoción representa también una motivación intrínseca que se ha asociado al empleo del estudiante de un enfoque de aprendizaje profundo y el empleo de estrategias de comprensión de la materia (De la Fuente et al., 2008), lo que permite explicar la influencia positiva de la orientación promoción en el rendimiento procedimental y global. En contextos deportivos también se constata que la motivación al éxito se relaciona con mayores niveles de rendimiento (García-Naviera \& Remor, 2011).

En cuanto a la posición del alumno en el aula, como ya se apuntó anteriormente, representa el autoconcepto del alumno en la materia. Se entiende que los alumnos que ocupan posiciones delanteras muestran mayor nivel de autoconfianza respecto a la materia y la metodología docente que aquellos que se sitúan en posiciones más atrasadas. Seguramente, esa autoconfianza es la que justifica la influencia de la posición del alumno en el aula sobre el rendimiento procedimental y global.

Estos resultados apuntan que el rendimiento procedimental requiere de la confluencia de motivación intrínseca y autoconfianza en la materia, lo que denota la implicación activa y la aplicación de estrategias de aprendizaje profundo por parte del alumno.

\section{Conclusiones e implicaciones}

El estudio realizado constata que el rendimiento conceptual difiere de manera significativa del rendimiento procedimental. En concreto, se constata que los alumnos analizados resultan más eficaces en la dimensión conceptual que en la procedimental. Se constata también que el conjunto de factores determinantes del rendimiento conceptual no coincide con aquellos determinantes del rendimiento procedimental. De todo esto se deduce que las dos dimensiones del rendimiento académico, lejos de resultar coincidentes, aluden a diferentes vertientes relacionadas con el aprendizaje adquirido por el alumno a lo largo de la asignatura. Se concluye, entonces, que el proceso de evaluación planteado en la asignatura atiende a distintas capacidades implicadas en el proceso de aprendizaje. Asimismo, la consideración diferenciada del rendimiento conceptual y procedimental permite un análisis detallado del resultado final del proceso de enseñanza-aprendizaje.

Por otro lado, se constata también que tanto las variables de presagio como las de proceso constituyen factores relevantes en la explicación del rendimiento académico de los alumnos. Específicamente, las variables presagio aportan una capacidad explicativa del $8 \%$ en el caso del rendimiento conceptual y en torno al $10 \%$ en el rendimiento procedimental y global. Los resultados indican que, independientemente del seguimiento de la asignatura, es más probable que los alumnos de género masculino más analíticos obtengan puntuaciones más altas en el rendimiento conceptual. Si además 
de lo anterior son alumnos con elevada motivación intrínseca (motivación promoción), se eleva su probabilidad de obtener puntuaciones altas tanto en el rendimiento procedimental como en el global. No obstante, estas variables son fijas -el alumno no tiene capacidad de influencia sobre ellas-, pero lo predisponen en su proceso de aprendizaje y, en consecuencia, en sus calificaciones. Por lo tanto, este resultado sirve como referencia a la hora de definir el perfil del alumno con mayores posibilidades en la aprehensión de la materia Inversiones Financieras analizada.

Por su parte, las variables de proceso (referidas al seguimiento que realiza el alumno de la materia) se relevan determinantes para los distintos tipos de rendimiento, pero sobre todo en la explicación del rendimiento global. En caso del rendimiento conceptual y procedimental su influencia, aunque significativa, es más escasa. Los resultados confirman que la asistencia a clase influye significativa y positivamente en el rendimiento académico en todas sus dimensiones. Además la posición del alumno en el aula, reflejo aproximado de su autoconfianza en la materia y afinidad con la metodología docente empleada, afecta decisivamente su rendimiento procedimental y global. En síntesis, aquellos alumnos que optan por situarse en las posiciones delanteras son los que obtienen mejores calificaciones. Se concluye entonces que el seguimiento de la materia resulta más asequible para alumnos de ciertas características personales, pero su implicación activa en el proceso de aprendizaje resulta determinante en sus resultados finales.

En suma, los resultados alcanzados apuntan diferencias notables entre el rendimiento conceptual y procedimental. En la muestra, los alumnos alcanzan mejores resultados en la dimensión conceptual y la selección de las variables explicativas más significativas aclara el motivo. Así, mientras el rendimiento conceptual depende de variables como el estilo cognitivo, el género y la asistencia, el procedimental requiere además de un enfoque de aprendizaje profundo que descansa en la capacidad de relacionar conceptos y trasladarlos a casos concretos.

Estos resultados aportan sugerencias interesantes para generar estrategias de intervención en los procesos de enseñanza-aprendizaje. Así, los resultados sugieren que el profesor debe fomentar la implicación del alumno en la materia, aunque sea la simple asistencia o la ubicación del alumno en posiciones delanteras del aula, puesto que eso estimulará el proceso de aprendizaje del alumno, lo que redundará en un mejor rendimiento académico, sobre todo en el rendimiento global. Además, el estudio constata que el rendimiento conceptual es el que menos se beneficia de la implicación del alumno en el seguimiento del proceso enseñanzaaprendizaje. Por tanto, a la hora de seleccionar las metodologías docentes, aquellas encaminadas al trabajo autónomo del alumno resultarán más apropiadas para contenidos conceptuales que para procedimentales. Para estos últimos parecen más apropiadas las relacionadas con trabajo en grupo, seminarios, estudios de casos, etc.

Sin embargo, los resultados obtenidos deben tomarse con la cautela propia que requieren las limitaciones del estudio. Entre estas destaca el reducido tamaño de la muestra, ya que puede afectar la potencia de los contrastes realizados. Además, sería interesante realizar esta investigación con una muestra más amplia que incluyera datos de otras asignaturas y la réplica en otros contextos de aprendizaje. Asimismo, resulta evidente que la representación de las variables de presagio y producto no es completa. No obstante, en este estudio el propósito no era tanto retratar con detalle el conjunto de variables que determinan el rendimiento académico, sino determinar la influencia diferencial de algunas variables determinantes sobre las distintas dimensiones del rendimiento académico. En sucesivas investigaciones se abordarán otras vertientes del modelo que permitan arrojar luz acerca de cuáles son los estudiantes a los que dirigir las actividades de motivación, en qué dirección y cómo evitar el fracaso académico.

\section{Referencias}

Allinson, C. W. \& Hayes, J. (1996). The cognitive style index: A measure of intuition-analysis for organizational research. Journal of Management Studies, 33(1), 119-135. 
Alonso, C. M., Gallego, D. L. \& Honey, P. (1994). Los estilos de aprendizaje. Procedimientos de diagnóstico y mejora. Bilbao: Ediciones Mensajero.

Alvarado, J. \& García Jiménez, M. V. (1997, septiembre). Incidencia de la asistencia a clase, del trabajo efectivo y de factores individuales sobre el rendimiento académico. Trabajo presentado en el V Congreso de Metodología de las Ciencias Humanas y Sociales, Sevilla, España.

Álvarez, V., García, E. \& Gil, J. (1999). El rendimiento académico en la universidad desde la perspectiva del alumnado. Revista Española de Orientación y Psicopedagogía, 17(10), 23-42.

Amelang, M. \& Bartussek, D. (1991). Psicología diferencial e investigación de la personalidad. Barcelona: Herder.

Arnold, M. J. \& Reynolds, K. E. (2009). Affect and retail shopping behavior: Understanding the role of mood regulation and regulatory focus. Journal of Retailing, 85(3), 308-320.

Bandura, A. (1987). Pensamiento y acción. Barcelona: Martínez Roca.

Bateman, T. S. \& Crant, J. M. (1993). The proactive component of organizational behavior: A measure and correlates. Journal of Organizational Behavior, 14(2), 103-118.

Beltrán, J. (1993). Procesos, estrategias y técnicas de aprendizaje. Madrid: Síntesis.

Biggs, J. B. (1988). Assessing study approaches to learning. Australian Psychologist, 23(2), 197-206.

Biggs, J. B. (2001). The reflective institution: Assuring and enhancing the quality of teaching and learning. Higher Education, 41(3), 221-238.

Brown, G. \& Atkins, M. (1988). Effective teaching in higher education. Londres: Routledge.

Cano, F. (2000). Diferencias de género en estrategias y estilos de aprendizaje. Psicothema, 12(3), 360-367.

Contreras-Torres, F. V., Espinosa-Méndez, J. C. \& Esguerra-Pérez, G. (2009). Personalidad y afrontamiento en estudiantes universitarios. Universitas Psychologica, 8(2), 311-322.

Cools, E. \& Van den Breeck, H. (2008). The hunt for the heffalump continues: Can trait and cognitive characteristics predict entrepreneurial orientation? Journal of Business Strategy, 18(2), 23-41.

Costa, P. T. \& McCrae, R. R. (1992). Revisited NEO Personality Inventory (NEO-PI-R) and NEO Five
Factor Inventory (NEO-FFI) professional manual. Odessa: Psychological Assessment Resources.

Costa, P. T. \& McCrae, R. R. (1999). Manual técnico del NEO-PI-R. Madrid: TEA.

Corno, L. \& Snow, R. E. (1986). Adapting teaching to individual differences among learners. En M. Wittrock (Ed.), Handbook of research on teaching (3a. ed., pp. 605-629). New York: McMillan.

Crant, J. M. (2000). Proactive behavior in organizations. Journal of Management, 26(3), 435-462.

De la Fuente, J., Martínez, J. M., Peralta, F. J. \& García, A. B. (2010). Percepción del proceso de enseñanzaaprendizaje y rendimiento académico en diferentes contextos instruccionales de la Educación Superior. Psicothema, 22(4), 806-812.

De la Fuente, J., Pichardo, M. C., Justicia, F. \& Berbén, A. (2008). Enfoques de aprendizaje, autorregulación y rendimiento en tres universidades europeas, Psicothema, 20(4), 705-711.

Echavarri, M., Godoy, J. C. \& Olaz, F. (2007). Diferencias de género en habilidades cognitivas y rendimiento académico en estudiantes universitarios. Universitas Psychologica, 6(2), 319-329.

Felman, L., Goncalves, L., Chacón-Puignau, G., Zaragoza, J., Bagés, N. \& de Pablo, J. (2008). Relaciones entre el estrés académico, apoyo social, salud mental y rendimiento académico en estudiantes universitarios venezolanos. Universitas Psychologica, 7(3), 739-751.

Fernández, E. (2001). ¿Cuál es el papel de alumnado dentro del proceso de enseñanza-aprendizaje en la Universidad? Revista de Educación, 325, 201-207.

García, M. V., Alvarado, J. M. \& Jiménez, A. (2000). La predicción del rendimiento académico: regresión lineal versus regresión logística. Psicothema, 12(2), 248-252.

García-Naviera, A. \& Remor, E. (2011). Motivación de logro, indicadores de competitividad y rendimiento en un equipo de jugadores de fútbol de competición varones entre 14 y 24 años. Universitas Psychologica, 10(2), 477-487.

González-Pienda, J., Núñez Pérez, J. C., Glez.-Pumariega, S. \& García, M. S. (1997). Autoconcepto, autoestima y aprendizaje escolar. Psicothema, 9(2), 271-289.

Higgins, E. T. (1998). Promotion and prevention: Regulatory focus as a motivational principle. Advances in Experimental Social Psychology, 30(1), 1-46. 
John, O. P., Caspi, A., Robins, R. W., Moffitt, T. E. \& Stouthamer-Loeber, M. (1994). The Little Five: Exploring the nomological network of the FiveFactor Model of personality in adolescent boys. Chid Development, 65(1), 160-178.

Maquilón, J. J. \& Hernández, F. (2011). Influencia de la motivación en el rendimiento académico de los estudiantes en formación profesional. Revista Electrónica Interuniversitaria de Formación del Profesorado, 14(1), 81-100.

Martín del Buey, F. \& Camarero Suárez, F. (2001). Diferencias de género en los procesos de aprendizaje en universitarios. Psicothema, 13(4), 598-604.

McClelland, D. C. (1989). Estudio de la motivación humana. Madrid: Lancea.

Navarro, Y. (2009). iInfluyen los rasgos de personalidad y el método docente empleado en al percepción del rendimiento académico del alumnado universitario? Revista Qurriculum, 22, 189-206.

Núñez Pérez, J., González-Pienda, J. A., García Rodríguez, M., González-Pumaniega, S., Roces Montero, C., Álvarez Pérez, L., et al. (1998). Estrategias de aprendizaje, autoconcepto y rendimiento académico. Psicothema, 10(1), 97-109.

Santana, L. E., Feliciano, L. A. \& Jiménez, A. B. (2009). Autoconcepto académico y toma de decisiones en el alumnado de bachillerato. Revista Española de Orientación y Psicopedagogía, 20(1), 61-75.

Santos, M. V., García, M. T. \& Vallelado, E. (2010, septiembre). La percepción directiva de distintos estímulos estratégicos. Trabajo presentado en el XX
Congreso Nacional de la Asociación Científica de Economía y Dirección de la Empresa, Granada, España.

Seibert, S. E., Kraimer, M. L. \& Crant, J. M. (2001). What do proactive people do? A longitudinal model linking proactive personality and career success. Personnel Psychology, 54(4), 845-874.

Severiens, S. E. \& Ten Dam, G. T. (1994). Gender differences in learning styles: A narrative review and quantitative meta-analysis. Higher Education, 27(4), 487-501.

Semin, G., Higgins, T., Gil de Montes, L., Estourget, Y. \& Valencia, J. F. (2005). Linguistic signatures of regulatory focus: How abstraction fits promotion more than prevention. Journal of Personality and Social Psychology, 89(1), 36-45.

Soto, G., Ferrándiz, C., Saínz, M., Ferrando, M., Prieto, M. D., Bermejo, D., et al. (2011). Características psicométricas del Cuestionario de Personalidad BFQ-NA. Aula Abierta, 39(1), 13-24.

Rodríguez, C. \& Herrera, L. (2009). Análisis correlacional-predictivo de la influencia de la asistencia a clase en el rendimiento académico universitario. Estudio de caso de una asignatura. Profesorado: Revista de Currículum y Formación del Profesorado, 13(2), 1-13.

Valle, A., González, R., Núñez, J. C. \& González, J. A. (1998). Variables cognitivo-motivacionales, enfoques de aprendizaje y rendimiento académico. Psicothema, 10(2), 393-412. 


\section{Maㅡ Valle Santos Álvarez, Eleuterio Vallelado}

\section{Apéndice}

Correlación entre variables explicativas

\begin{tabular}{lcccccc}
\hline & CSI & PM & SEX & EXT & ASIST & POSIC \\
\hline CSI & 1 & -0.012 & 0.115 & -0.137 & 0.034 & -0.029 \\
PM & 1 & -0.062 & 0.19 & 0.03 & 0.15 \\
SEX & & 1 & 0.149 & 0.181 & -0.137 \\
EXT & & & 1 & -0.021 & -0.007 \\
ASIST & & & & 1 & -0.09 \\
POSIC & & & & & 1 \\
\hline
\end{tabular}

$* p<0.05 . * * p<0.01$.

Fuente: elaboración propia. 